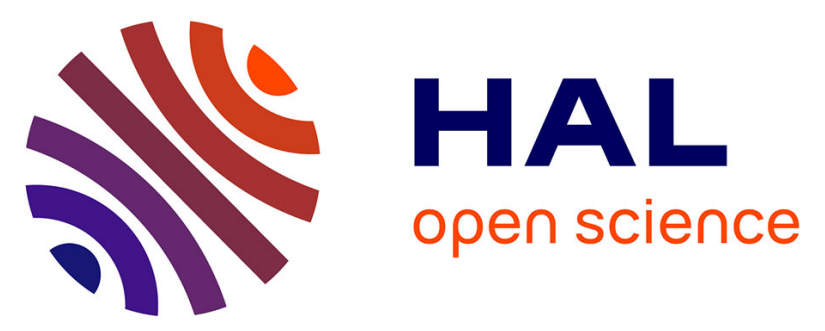

\title{
Towards an EDSL to enhance good modelling practice for non-linear stochastic discrete dynamical models Application to plant growth models
} Benoit Bayol, Yuting Chen, Paul-Henry Cournède

\section{- To cite this version:}

Benoit Bayol, Yuting Chen, Paul-Henry Cournède. Towards an EDSL to enhance good modelling practice for non-linear stochastic discrete dynamical models Application to plant growth models. SIMULTECH 2013, Jul 2013, Iceland. pp.132-138, 10.5220/0004481101320138 . hal-01001669

HAL Id: hal-01001669

https://hal-centralesupelec.archives-ouvertes.fr/hal-01001669

Submitted on 4 Jun 2014

HAL is a multi-disciplinary open access archive for the deposit and dissemination of scientific research documents, whether they are published or not. The documents may come from teaching and research institutions in France or abroad, or from public or private research centers.
L'archive ouverte pluridisciplinaire HAL, est destinée au dépôt et à la diffusion de documents scientifiques de niveau recherche, publiés ou non, émanant des établissements d'enseignement et de recherche français ou étrangers, des laboratoires publics ou privés. 


\title{
Towards an EDSL to enhance good modelling practice for non-linear stochastic discrete dynamical models Application to plant growth models
}

\author{
Benoit Bayol $^{1}$, Yuting Chen ${ }^{1}$ and Paul-Henry Cournède ${ }^{1}$ \\ ${ }^{1}$ Ecole Centrale Paris, Laboratory of Applied Mathematics and Systems, 92290, Châtenay-Malabry, France \\ \{benoit.bayol,yuting.chen, paul-henry.cournede\}@ecp.fr
}

\begin{abstract}
Keywords: general state-space model, grid simulation, parallelism, parameter estimation, filtering, sensitivity analysis, uncertainty analysis, model selection, data assimilation, multi-paradigm programming, EDSL

Abstract: A computational formalism is presented that structures a $\mathrm{C}++$ library which aims at the modelling, simulation and statistical analysis of stochastic non-linear discrete dynamical system models. Applications concern the development and analysis of general plant growth models.
\end{abstract}

\section{INTRODUCTION}

With the increasing need in modelling in all fields of science, and sometimes a lack of precautions in the way models are developed and used, some authors tried to define and promote good modelling practices for environmental sciences (Van Waveren et al., 1999) or in physiology and medicine (Carson and Cobelli, 2001) by proposing different steps in the modelling process, from conceptual work to model applications. These steps include the use of statistical analysis tools like parameter estimation, sensitivity analysis, uncertainty analysis or model selection.

Some existing numerical platforms like R, Scilab or Matlab propose existing algorithms belonging to these categories but no real standardization for the inputs and outputs of these tools. Moreover some existing modelling platforms like Modelica, Xcos or Simulink have a good modelling framework but are mainly deterministic and stream oriented which prevents implementing powerful estimation methods in a proper statistical framework. Finally all these platforms are disconnected in the sense that going from one to another for analyzing a model is not an easy task in terms of engineering. Each tools and algorithms have a different set of required inputs.

In this context, our objective is to design a single library that allows to create, to evaluate and to analyze models with a common language among modellers and statisticians. This library has the following characteristics :

- it uses a multi-paradigm programming style with emphasis on generic and functional paradigms.

- it uses a common syntax for modelling, simulation and analysis algorithms.

- it provides a flexible template for model observations, adapted to the heterogeneous and irregular observations of biological systems.

- it is adapted to stochastic systems, particularly the implementation of modelling and observations noises.

- implements statistical methods for model analysis and evaluation

In section 2, we describe the modelling framework of the platform. In section 3, we show how to represent the simulation framework with a projection on a $3 \mathrm{D}$-grid that eases the implementation of numerical methods and their parallel computation. In section 4, we present an overview of the implemented methods. In section 5, we illustrate the potentials of the library on a complex test case of data assimilation of a plant model. Finally, we will discuss about the perspectives of this library.

\section{MODELLING}

Better understanding of plant development and growth is a key issue to make agriculture practice more competitive and more respectful of the environment.

Agronomic researchers and engineers have built several models for this purpose. Geometrical or empir- 
ical at first, models are more and more mechanistic, with the development of agro-environmental ((Brisson et al., 2003)), and functional-structural models ((de Reffye et al., 2008), (Vos et al., 2010)) which are used for the description at macro and mesoscale levels.

These mechanistic models have the following characteristics:

- complexity in terms of the numbers of interacting processes and of parameters.

- difficult parameter estimation due to the nonlinearity of the model and irregularity of data.

- sophisticated and costly methods for their analysis.

- possibly high memory need during computation for models of plants with complex structures like trees.

A general representation of plant growth can be given in the general state-space form, with model equations describing the discrete evolution of the state variables $X \in \mathbb{R}^{x}$ across time steps, and observation equations, giving the system observation variables $X \in \mathbb{R}^{y}$ as functions of the state variables (Cournède et al., 2011). For biological systems, these observation functions may be very rare (no observation at most time steps) and heterogeneous (different types of observations at different observation times).

Let us decompose the observation vectors $Y$ in $k$ elementary sub-vectors $Y=\left(Y_{1}, Y_{2}, \ldots, Y_{k}\right)$, such that at each step of system observation $j$, the observation function $G_{j}$ can be described by a subset of the $\left\{Y_{i}\right\}_{1 \leq i \leq k}$, corresponding to the set of variables that are observed at step $j$. The elementary sub-vectors $Y_{i}$ are called observers (their choice is not unique) and $\tau_{i}=\left\{j\right.$ such that $Y_{i}$ is observed at step $\left.j\right\}$ is called the timeline of observer $i$.

Equation (1) describes the general state-space form taking into account the irregularity and heterogeneity of data.

$$
\left\{\begin{array}{l}
X_{n+1}=F_{n}\left(X_{n}, U_{n}, P, \varepsilon_{n}\right) \\
Y_{n}=G_{n}\left(X_{n}, P, \varepsilon_{n}^{\prime}\right)=\left(Y_{i} \Lambda_{\tau_{i}}(n)\right)_{1 \leq i \leq k}
\end{array}\right.
$$

with $\Lambda_{\tau_{i}}(n)=1$ if $n \in \tau_{i}$, else 0 .

In order to translate Equation (1) into an effective code for simulation, we first give the following definitions:

- A dynamical model denotes a 6-tuple $\mathcal{M}=$ $\left\{X, U, P, \varepsilon_{\mathcal{M}}, I N I T, N E X T\right\}$ where:

- $\{X, U, P\}$ denote respectively the set of state variables, the set of control variables, the set of parameters of the full model.
- $\varepsilon_{\mathcal{M}}$ denotes the set of stochastic variables of the model errors. The space dimension corresponds to the dimension of the random vector used in the model equations.

- INIT denotes an initialization function to determine the initial state $X^{0}$ such as $X^{0}=I N I T(P)$ and INIT $: X \times P \rightarrow X$

- NEXT represents the transition function of the dynamical model such as NEXT:X $\times U \times P \times$ $\varepsilon_{\mathscr{M}} \rightarrow X$

- An observation model denotes a 5-tuple $O=$ $\left\{X, P, Y, \varepsilon_{O}, O B S E R V E\right\}$ where:

- $Y$ denotes the output of the observation model.

- $\varepsilon_{O}$ denotes the set of stochastic variables for the observation errors.

- OBSERVE denotes an observation function such as $O B S E R V E: X \times P \times \varepsilon_{O} \rightarrow Y$

- An observer denotes a 2-tuple $O=\{O, T M L\}$ where $T M L$ is a timeline which controls the observation of the dynamical system.

Thus the global stochastic dynamic system model (with observations) is denoted by a 8-tuple $S=\left\{X, U, P, Y, \varepsilon_{\mathcal{M}}+\right.$ $\left.\left\{\varepsilon_{O}\right\}_{1 \leq i \leq k}, I N I T, N E X T,\{\text { OBSERVE,TML }\}_{1 \leq i \leq k}\right\}$ where:

- the indexes $i, 1 \leq i \leq k$ represent different observers. We do not consider a unique observer because of the irregularity and diversity of observed variables. It is a very important specificity of biological systems for which experiments are difficult or costly (Cournède et al., 2011). The error models for each observer will also be specific.

- $\varepsilon_{\mathcal{M}}+\left\{\varepsilon_{O}\right\}_{1 \leq i \leq k}$ represents the total set of stochastic vectors for model simulation.

To generate the random sequences $\left(\varepsilon_{n}\right)_{0 \leq n \leq N-1} \in$ $\left(\varepsilon_{\mathcal{M}}\right)^{N}$, with $N$ the maximum simulation time and $\left(\varepsilon_{n}^{\prime}\right)_{0 \leq n \leq N-1} \in\left(\varepsilon_{O}\right)^{N}$, for each of the $k$ different observers, we also define a random vector model by a 3-tuple $\mathcal{V}=\left\{P, \varepsilon_{\mathcal{V}}, L A W\right\}$ such as:

- $P$ denotes the set of parameters of the $L A W$.

- $\varepsilon_{\mathcal{V}}$ is the set of stochastic vectors

- $L A W$ represents the law of the probability distribution such as $L A W: P \times[0 ; 1]^{v} \rightarrow \varepsilon_{\mathcal{V}}$, where $v$ is the dimension of the random vector: if $\varepsilon_{\mathcal{V}} \subset \mathbb{R}^{v}$ is a probability space of dimension $v$, there exists a bijection $\psi$ from $[0 ; 1]^{v}$ onto $\varepsilon_{\mathcal{V}}$ given by the inverse of the marginal cumulative distribution function of each component.

A simulation of the random vector is thus built from a random model $\mathcal{V}$ and a generator that generates a 
sequence in $[0 ; 1]^{v}$. Different types of generators exist (pseudo-random based on congruential sequences for example as Mersenne-Twister (Matsumoto and Nishimura, 1998), quasi-random (Kocis and Whiten, 1997), ...). The sequence generated by the generator is usually uniquely determined by a seed, corresponding to the first element of the sequence in $[0 ; 1]^{v}$. Therefore, a simulation of the random vector is a 5-tuple $\left\{\mathcal{V}, p, N, S_{0}, G E N E R A T E\right\}$ where $S_{0}$ is the seed of generator and GENERATE the function that generate the random sequence in $[0 ; 1]^{\nu}, p \in P$ and $N$ the maximal time of the simulation.

Such random variable simulation is used in the dynamical system simulation to generate both model and observation noises (for each of the $k$ observers).

\section{SIMULATION}

In this section we detail how the modelling framework can be projected onto a 'simulation grid' to categorize and formalize the different algorithms used for model analysis. This also helps to consider the transition to parallel computing. The categorization will be conducted both in terms of input arguments of the algorithms and pathways.

We give the following definitions:

- a context $c$ denotes the initial conditions and associated control variables. In our case, the control variables are given by the environmental conditions and are supposed to be fully known at the beginning of the simulation. Therefore $c$ is composed of $X^{0}$ and $\left(U_{n}\right)_{0 \leq n \leq N}$, where $N$ represents the last time step of the simulation.

- parameters $p$ are the full vector of parameters of the observation model and dynamical model.

- an observer list $[o]$ denotes the composition of several observers i.e. a several observation functions with their timelines.

- a list of seed [seed] is given to either dynamical model or observation model for initializing the random generators.

- an observation list $y$ denotes the result of observers during a simulation

- a simulation is the combination of a dynamical model, an observer list, a context and a set of parameters

- simulate denotes a function that applies a parameters set to a list of simulations.

Left of the figure 1 summarizes all these concepts in a syntax tree.

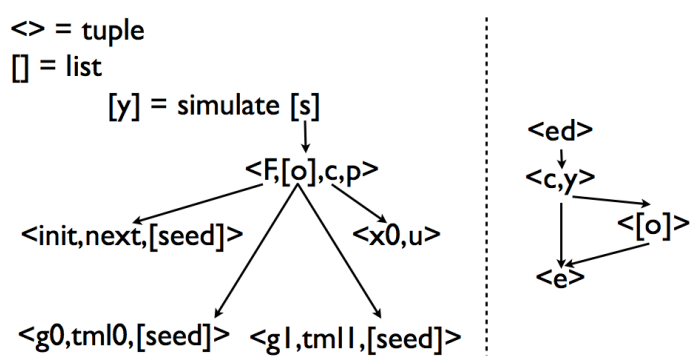

Figure 1: Simulation syntax tree.

Moreover a key feature for interacting with users is to rebuild simulations from experimental data by extracting the list of context and observer list. For this purpose we define:

- an experimental data ed denotes data that have been observed for a given context.

- experiment $e$ denotes observations that will be observed for a given context. Experiment is a tuple composed of a context and an observer list.

Right of the figure 1 summarizes these two concepts. We have thus defined data structures for representing observation data and simulations, and a simulation function.

As we will see in section 4 most algorithms use a combination of contexts and parameters as inputs. As a result, these algorithms manipulate lists of simulation.

A conceptual grid helps to classify the algorithms of interest for model analysis and estimation detailed in section 4 . The classification is done regarding which part of the grid is used and how we go through it.

For the sake of clarity, we leave aside the notion of observer and use only context and parameters.

Algorithms in section 4 have to simulate a list of simulations by the combination of:

- a lot of contexts for a given parameter set.

- a single context with a lot of parameter sets.

- a lot of contexts with a lot of parameter sets.

On figure 2 we give a representation of the simulation grid, with the following axis:

- $\mathrm{x}$-axis for contexts.

- $y$-axis for parameter sets.

- z-axis for time steps.

For each triple $(x, y, z)$ we associate an equivalent triple $\left(C_{i}, P^{j}, n\right)$ and its transformation $\left[Y_{n}\right]_{i}^{j}$ through the simulation function which is the result of doing observations at time $n$ on cell $(i, j)$.

There are two ways for going through the grid: 


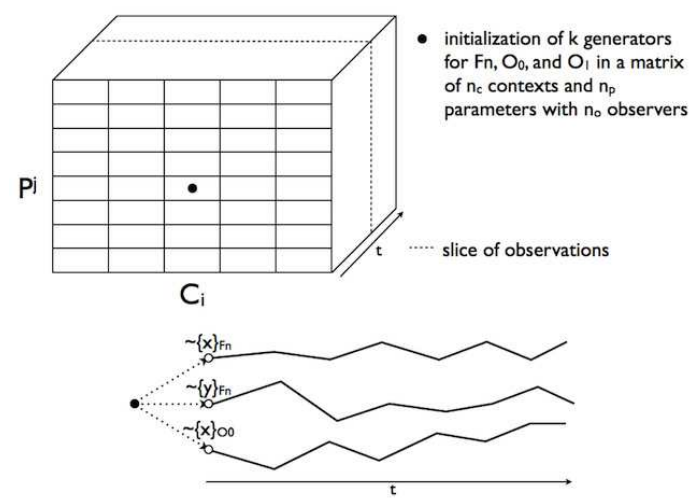

Figure 2: Grid for simulation.

- in one single run which corresponds to obtaining a full observation vector for all simulations.

- per step which corresponds to obtaining a slice of observations as shown in figure 2.

Both model and observation equations might be perturbed by noises. There is a unique random generator for each noise, that is to say for each stochastic variable.

In figure 2 each cell of the plane (corresponding to one context and one parameter set) has its own generator list denoted by a black dot. This black point is composed of several dots which are the seed of each random generator. $\sim\left\{\varepsilon_{\mathscr{M}}\right\}$ is the generator associated to the model noises and $\sim\left\{\left(\varepsilon_{O}\right)_{i}\right\}$ is the generator associated to the observer $O_{i}$, for all $i, 1 \leq i \leq k$. The curves illustrate the random trajectories.

\section{ANALYSIS}

Key steps in the modelling process concern analysis and parameter estimation.

The following methods are implemented in the platform:

- frequentist parameter estimation approaches, like generalized least squares estimator (GLSE) or maximum likelihood estimator (MLE). Generally, the estimation involves a context list and an observer list, and handles at each algorithmic step a single parameter set. It is not the case however, for Monte-Carlo methods such as stochastic expectationmaximization (see (Trevezas and Cournède, 2013) in the context of plant growth). The equivalent function signature with previous notations is frequentist_parameter_estimation $(<$ $M,[e d], p>)$. We only go through the first row of the grid to compute all observation lists $[Y]_{0}^{i}$ with only one parameter set. Then we compare all these lists to the experimental one and select another parameter set to minimize this distance.

- Bayesian inference approaches based on filtering methods, like convolution particle filter (Chen and Cournède, 2012) or unscented kalman filter (Julier et al., 2000), take a single context and an observer list with a list of parameter sets. The equivalent function signature is filtering_parameter_estimation $(<M, e d,[p]>$ ) . We only go through the first column of the grid and stop at each observation time step to implement the filtering process based on the experimental data: at each observation step, the parameters and states are updated for all the selected simulations. The idea is to provide estimations with reliable uncertainty that are appropriately assessed with the population of parameter sets. A more detailed presentation of convolution particle filter can be found in 5 .

- sensitivity analysis approaches, like standard regression coefficient or Sobol (Saltelli et al., 2008) (Wu et al., 2012), take a single context and an observer list with a list of parameter sets. The equivalent function signature with previous notation is sensitivity_analysis $(<M, e,[p]>)$. The way of going trough the grid is slightly the same as for filtering parameter estimation methods. The exception concerns the different triggers. In sensitivity analysis parameters are not changed during the algorithm.

- uncertainty analysis approaches, using Monte Carlo samples or the unscented transform (Julier et al., 2000), follow the same rules as sensitivity analysis. The equivalent function signature is uncertainty_analysis $(<M, e,[p]>)$.

- model selection (Baey et al., 2012) computes criterion like mean square prediction error or Akaike information criterion for a list of models. A criterion follows the signature model_selection $(<$ $M,[e d], p>)$ but the general method will use a list of models $[M]$ for making comparison among the criterion results.

\section{TEST CASE}

In this section we describe the context of convolution particle filtering and its characteristics. Previous elements are not specifically related to plant growth models. In this test case, we consider the param estimation of a plant growth model. Some specific characteristics have to be taken in account. 
As said in section 1 plant growth models are generally characterized by complex interacting processes and a great number of model parameters. Moreover experimental data acquisition tends to be costly (experiments in fields), inaccurate (coming from satellite images), and irregular (sometimes observations can not be done). Thus the parametrization of these models is a key issue which may affect the quality of model prediction. Therefore we use the convolution particle filter method. (Campillo and Rossi, 2009) and an adaptation developed by (Chen et al., 2012).

The objective of this method is to estimate jointly the parameters and the hidden states of the system from online data i.e. data that comes from time to time improving the database. This kind of technique is known as data assimilation. The idea is to sample M particles (i.e. a parameter set and a state) and to propagate them through the model until the next available measurement to compare with the predicted states. Then we compute for each particle a weight, according to experimental data and prediction, which helps to classify and select the best particles that are closer to the real experimental case.

The following results are based on the CPF method applied to the Log Normal Allocation and Senescence (LNAS) daily crop model with real experimental data (Chen et al., 2013). The equations of the LNAS model are derived for sugar beet with three main processes during the plant growth period: biomass production, allocation and senescence. Two compartments are taken into account: foliage and root system.

Based on the parameter estimation results from the 2010 dataset with an iterative version of the CPF method, we conducted the data assimilation approach with the CPF algorithm by recalibration the parameters and readjusting the hidden states of interest based on the data of early growth stage (five first data) of the 2006 dataset. The predictive capacity of the model for the last two dates of measurements is compared in two cases: with data assimilation and from pure uncertainty analysis (based on the calibration result, propagation of the uncertainty with the 2006 context). In our example we consider data collected at 12 different dates in 2010 for the calibration step: $O_{2010}=$ $\{54,68,76,83,98,104,110,118,125,132,145,160\}$. We also have 7 measurement dates in 2006: $O_{2006}=\{54,59,66,88,114,142,198\}$

Figure 3 illustrates the prediction results given by the two cases. In the case of data assimilation with CPF, not only the point predictions are more accurate, but the related uncertainty is also reduced. On the contrary, the predicted confidence interval given by uncertainty analysis (without data assimilation) does not even contain the real measurements (last two points).

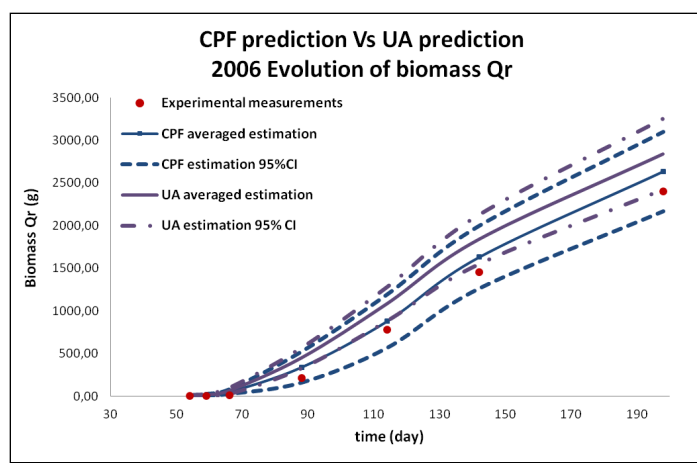

Figure 3: Comparison of CPF Data Assimilation with Monte Carlo UA method

This result clearly suggests an obvious advantage of the CPF data assimilation method in terms of prediction capacity.

However such method is rather time and memory consuming since for a run with 40000 particles we need $8 \mathrm{~Gb}$ of RAM and 18 hours of computation for a sequential job. The advantage and feasibility of parallel computation is obvious with the formalization as a simulation grid, and we are currently working on the implementation. Of course, the computation time depends also of the analyzed model.

\section{DISCUSSION}

We have shown the basic principles that structure our library and how they are related to the domain of application, discrete nonlinear, stochastic models, with potentially heterogeneous or rare observations, with the example of plant growth. There are still some steps to fulfill in order to call it a domain-specific language.

\subsection{TOWARDS AN EMBEDDED DOMAIN SPECIFIC LANGUAGE}

An embedded domain specific language is a language hosted into another one with a semantic dedicated to a domain. It has the characteristic to exploit the host language syntax which helps to focus on domainspecific question and reduce maintenance.

The choice of $\mathrm{C}++$ has been made in regards of :

- developers skills inside the project

- the number of reliable libraries in the community like Boost, MKL or Armadillo

- the philosophy of "abstractions that do not impose space or time overheads" (Stroustrup, 2012) 
- its performance compared to other existing languages (Hundt, 2011)

Our current work is to formalize the ideas that have been developed in section 2 and section 3 by defining the abstract syntax tree and inference rules.

Genericity of the library is an important goal, especially the ability to analyze any model that can be formulated by equation 1 .

We use templates because of its capacity of emulating structural sub-typing and by experience this kind of sub-typing is more convenient to our activity than nominal sub-typing with the original inheritance mechanism of object-oriented programming. Moreover this orientation could allow us to use structures or tuples in conjunction with Vexcl or Thrust libraries in an easy way through tag dispatching technique.

\subsection{WORKFLOW}

This framework was built with constant exchanges between the modellers, the mathematicians developing the methods, and software engineers. It helped us to understand the domain of course but also the way we were working on this domain. Most of the time a given model is associated to a given modeller and the transmission and integration in terms of code is quite complex if it does not follow a strict interface. Therefore we have defined a terminology and tool for managing this workflow. The tool is developed in python and is inspired by management tool frequently available with web framework like Rails or Symfony. The platform itself cannot be seen without its managing tool in order to establish a way of communication during the development of models and methods.

\subsection{CONCLUSION}

The above formalism has been designed with a bottom-up approach and is used in our team for the implementation of our tools. It unifies our thinking about modelling, simulation and analysis.

We did not linked yet our work to existing formalism like DEVS, stochastic petri nets, P-DEVS, picalculus. We expect to find a way for the support of concurrency models for biological systems like plant-soil interaction by looking at DEVS/P-DEVS and DESS. (Zeigler et al., 1995)

In the long term we believe that that a DSL can be derived from our EDSL for delivering a GUI tool to end-users.

\section{REFERENCES}

Baey, C., Didier, A., Li, S., Lemaire, S., Maupas, F., and Cournède, P.-H. (2012). Evaluation of the Predictive Capacity of Five Plant Growth Models for Sugar Beet. In Kang, M., Dumont, Y., and Guo, Y., editors, Plant Growth Modeling, Simulation, Visualization and Applications - PMA12, pages 30-37, Shanghai, China. IEEE.

Brisson, N., Gary, C., Justes, E., Roche, R., Mary, B., Ripoche, D., Zimmer, D., Sierra, J., Bertuzzi, P., Burger, P., Bussiére, F., Cabidoche, Y., Cellier, P., Debaeke, P., Gaudillére, J., Hènault, C., Maraux, F., Seguin, B., and Sinoquet, H. (2003). An overview of the crop model STICS. European Journal of Agronomy, 18:309-332.

Campillo, F. and Rossi, V. (2009). Convolution Particle Filter for Parameter Estimation in General State-Space Models. IEEE Transactions in Aerospace and Electronics., 45(3):1063-1072.

Carson, E. and Cobelli, C. (2001). Modelling Methodology for Physiology and Medicine. Academic Press, San Diego (US).

Chen, Y., Bayol, B., Loi, C., Trevezas, S., and Cournède, P.H. (2012). Filtrage par noyaux de convolution itératif. In Actes des 44émes Journèes de Statistique JDS2012, Bruxelles 21-25 Mai 2012.

Chen, Y. and Cournède, P.-H. (2012). Assessment of parameter uncertainty in plant growth model identification. In Kang, M., Dumont, Y., and Guo, Y., editors, Plant growth Modeling, simulation, visualization and their Applications (PMA12). IEEE Computer Society (Los Alamitos, California).

Chen, Y., Trevezas, S., and Cournède, P.-H. (2013). Iterative convolution particle filtering for nonlinear parameter estimation and data assimilation with application to crop yield prediction. In Society for Industrial and Applied Mathematics (SIAM): Control \& its Applications, San Diego, USA.

Cournède, P.-H., Letort, V., Mathieu, A., Kang, M.-Z., Lemaire, S., Trevezas, S., Houllier, F., and de Reffye, P. (2011). Some parameter estimation issues in functional-structural plant modelling. Mathematical Modelling of Natural Phenomena, 6(2):133-159.

de Reffye, P., Heuvelink, E., Barthélémy, D., and Cournède, P.-H. (2008). Plant growth models. In Jorgensen, S. and Fath, B., editors, Ecological Models. Vol. 4 of Encyclopedia of Ecology (5 volumes), pages 2824-2837. Elsevier, Oxford.

Hundt, R. (2011). Loop recognition in c++/java/go/scala In Proceedings of Scala Days 2011.

Julier, S., Uhlmann, J., and Durrant-Whyte, H. (2000). A new method for the nonlinear transformation of means and covariances in filters and estimators. IEEE Transactions on Automatic Control, 45(3):477-482.

Kocis, L. and Whiten, W. J. (1997). Computational investigations of low-discrepancy sequences. ACM Trans. Math. Softw., 23(2):266-294.

Matsumoto, M. and Nishimura, T. (1998). Mersenne twister: a 623-dimensionally equidistributed uni- 
form pseudo-random number generator. ACM Trans. Model. Comput. Simul., 8(1):3-30.

Saltelli, A., Ratto, M., Andres, T., Campolongo, F., Cariboni, J., Gatelli, D., Saisana, M., and Tarantola, S. (2008). Global Sensitivity Analysis. John Wiley\&Sons, the primer edition.

Stroustrup, B. (2012). Foundations of c++. In Seidl, H., editor, Programming Languages and Systems, volume 7211 of Lecture Notes in Computer Science, pages 125. Springer Berlin Heidelberg.

Trevezas, S. and Cournède, P.-H. (2013). A sequential monte carlo approach for mle in a plant growth model. Journal of Agricultural, Biological, and Environmental Statistics, In press.

Van Waveren, R., Groot, S., Scholten, H., Van Geer, F., Wosten, H., Koeze, R., and Noort, J. (1999). Good modelling practice handbook. Technical Report 9905, STOWA, Utrecht, RWS-RIZA, Lelystad, The Netherlands.

Vos, J., Evers, J. B., Buck-Sorlin, G. H., Andrieu, B., Chelle, M., and de Visser, P. H. B. (2010). Functionalstructural plant modelling: a new versatile tool in crop science. Journal of Experimental Botany, 61(8):21012115.

Wu, Q., Cournède, P.-H., and Mathieu, A. (2012). An efficient computational method for global sensitivity analysis and its application to tree growth modelling. Reliability Engineering \& System Safety, 107:35-43.

Zeigler, B., Song, H. S., Kim, T. G., and Praehofer, H. (1995). Devs framework for modelling, simulation, analysis, and design of hybrid systems. In In Proceedings of HSAC, pages 529-551. Springer-Verlag. 